\title{
Applying balancing techniques in traffic sign recognition
}

\author{
Sheila Esmeralda Gonzalez-Reyna ${ }^{* 1}$, J. Fco. Martinez-Trinidad ${ }^{2}$, J. Ariel Carrasco-Ochoa ${ }^{2}$, J. Gabriel \\ Avina-Cervantes ${ }^{1}$, Sergio Ledesma-Orozco ${ }^{1}$ \\ ${ }^{1}$ Universidad de Guanajuato, División de Ingenierías Campus Irapuato, Salamanca, Gto. Mexico \\ ${ }^{2}$ Instituto Nacional de Astrofisica Optica y Electronica, Computer Science Department,Puebla, Mexico
}

Received: September 3, 2014

DOI: $10.5430 /$ air.v3n4p38
Accepted: September 23, 2014 Online Published: October 24, 2014

URL: http://dx.doi.org/10.5430/air.v3n4p38

\begin{abstract}
Traffic Sign Recognition systems aim to determine the meaning of traffic signs in highways for real-world applications such as traffic sign inventory or driver assistance systems. Traffic sign datasets are inherently imbalanced, i.e. some traffic signs appear more frequently than others. One serious consequence of this imbalance is the low recognition rates of minority classes (classes with fewer training cases). In this paper, we propose a new method for improving traffic sign recognition of minority classes, by applying balancing algorithms. As a result, our proposed method improves minority class recognition rates up to $28 \%$ compared to traditional methods.
\end{abstract}

Key Words: Traffic sign recognition, Balancing algorithms, Normalized grayscale information, Histograms of oriented gradients

\section{Introduction}

Traffic Sign Recognition (TSR) consists in automatically identifying traffic signs for driver assistance systems or traffic sign inventory applications. However, some traffic signs like speed limits are more frequent than others, for example deer warnings, because some traffic signs depend on the environment. Hence, traffic sign datasets are inherently imbalanced. Traditional pattern recognition tasks for imbalanced datasets present unfavorable recognition results for minority classes. This can be caused by the lack of information needed to represent minority classes. Although in the literature some balancing algorithms ${ }^{[1,2]}$ have been proposed to deal with imbalance problems, none of them have ever been applied to traffic sign recognition. Therefore, in this paper, we propose a new method for traffic sign recognition that applies balancing algorithms, in order to increase the recognition rates of minority classes. This paper is organized as follows. Some existing Traffic Sign Recognition systems are described in Section 2. Our proposed method is introduced in Section 3. Section 4 shows and analyzes experimental results. Finally, Section 5 gives final conclusions and directions for future work.

\section{Related work}

TSR has been addressed in many different ways. ${ }^{[3]}$ TSR is commonly treated as a two-stage problem: detection and recognition. Detection aims to localize traffic signs immersed in a highway scene, ${ }^{[4]}$ while recognition intents to

\footnotetext{
*Correspondence: Sheila Esmeralda Gonzalez-Reyna; Email: sheila.esmeralda.gonzalez@gmail.com; Address: Universidad de Guanajuato. División de Ingenierías Campus Irapuato-Salamanca. Carretera Salamanca-Valle de Santiago km. 3.5+1.8km. Comunidad de Palo Blanco, C.P. 36885. Salamanca, Gto. Mexico.
} 
identify the previously detected signs. ${ }^{[5]}$ In this paper, we are only focused in recognition.

In TSR, as in other recognition tasks, the choose of suitable features is important because supervised classifiers can perform better when they use appropriate information. Histograms of Oriented Gradients (HOG), proposed by Dalal and Triggs, ${ }^{[6]}$ have been successfully applied to TSR. Overett and Petersson ${ }^{[7]}$ proposed two modifications to HOG features named LiteHOG+ and Single Bin HOG (SHOG) to characterize and classify traffic signs using LogitBoost. Zaklouta, Stanciulescu, \& Hamdoun ${ }^{[8]}$ and Stallkamp, Schipsing, Salmen, \& Igel, ${ }^{[5]}$ also used HOG features, jointly with Linear Discriminant Analysis (LDA). ${ }^{[9]}$ Random forests and k-d trees performed the classification in Ref.8 while nearest neighbor classifier was applied in Ref.5.

Other popular features used for TSR are grayscale information. Maldonado-Bascon et al. ${ }^{[23]}$ used normalized grayscale information to classify traffic signs with Support Vector Machines (SVM). Ciresan, Meier, Masci, \& Schmidhuber ${ }^{[11]}$ and Sermanet $\&$ LeCun ${ }^{[12]}$ used different grayscale and color normalized features, and classified using Convolutional Neural Networks (CNN).

One interesting characteristic of the TSR methods reported in the literature, is that they are composed by two steps: 1) features generation and 2) classification. Our main contribution is the addition of another step, where a balancing algorithm is applied to the traditional TSR steps in order to overcome the negative consequences of an imbalanced training set on classification tasks.

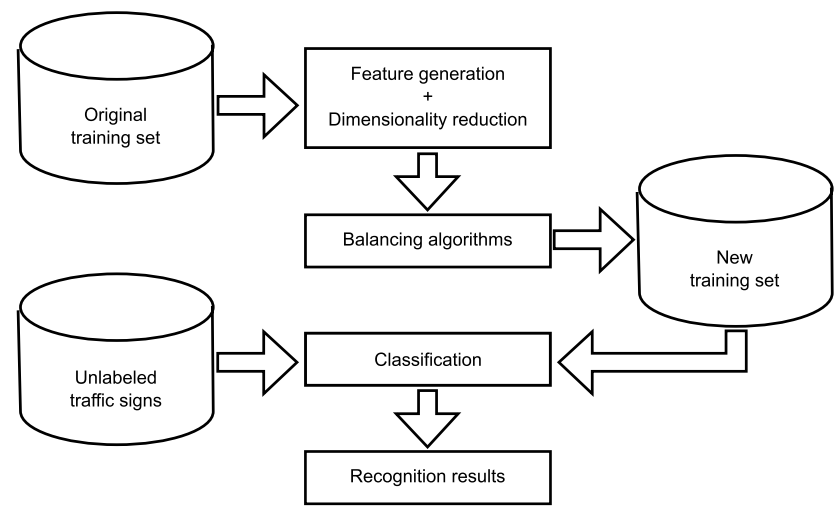

Figure 1: Proposed method

\section{Proposed method}

A training set is imbalanced when it contains many training cases of some classes (majority classes), but just a few in other classes (minority classes). In traffic sign recognition, some signs are more frequent than others and, as a consequence, traffic sign datasets are imbalanced. One important consequence of imbalanced training sets is the poor classification rates in minority classes, while majority classes tend to be accurately recognized.

Published by Sciedu Press
There are two approaches for improving classification accuracies on imbalanced training sets: ${ }^{[1]}$ balancing datasets by either resampling minority classes ${ }^{[13]}$ or subsampling majority classes; ${ }^{[10,14,15]}$ and implementing cost-sensitive classifiers. ${ }^{[16,17]}$ However, cost-sensitive classifiers may not be useful on this particular problem, because relative costs for different traffic signs could be difficult to determine. Therefore for solving imbalance in TSR we propose to apply balancing algorithms.

One common balancing algorithm consists in randomly oversampling minority clases in order to produce a balanced training set ${ }^{[18]}$ we will refer to this algorithm as RES. Chawla, Bowyer, Hall, \& Kegelmeyer ${ }^{[13]}$ proposed a variation of this algorithm named SMOTE, where minority classes are oversampled by creating new synthetic training cases, using a combination of the available nearest neighbors in those classes.

Balancing algorithms have the main purpose of reducing the imbalance ratio. Imbalance ratio is defined as the number of instances in the majority class, divided by the number of instances in the minority class. ${ }^{[2,19]}$

The initial step in many recognition systems, as in our proposed method, consists in obtaining an appropriate feature set. In many cases, these feature sets may be composed by a large amount of attributes, and then, it may be advisable to apply a dimensionality reduction algorithm in order to simplify the training set.

Considering that in TSR the training set is imbalanced, we propose, as a second step, the use of balancing algorithms in order to increase the amount of cases in the minority classes. The more information is available in the class, the more probability to accurately labeling new cases. Finally, once the new training set has been generated through the application of a balancing algorithm, the next step is training a classifier using this new training set. Classifiers perform better when an appropriate set of features is chosen, and when a suitable amount of information per class is available.

Figure 1 shows our proposed method diagram. The original imbalanced training set is processed to obtain a suitable set of features. Then, if the set of features has too many attributes, dimensionality reduction shall be applied. Finally, a balancing algorithm is applied to reduce the imbalance ratio, generating a more suitable training set for training a classifier.

\section{Experimental results}

This section presents a set of experiments performed in order to demonstrate that the proposed method improves the recognition rate of minority classes in TSR. 


\subsection{Database}

Our proposed method was tested using a popular dataset for TSR, the German Traffic Sign Recognition Benchmark (GTSRB). ${ }^{[5]}$ This dataset was designed with the purpose of classifying traffic signs, i.e. it is composed only by traffic sign images.

The GTSRB dataset is composed by 39,209 images for training, and 13,630 for validation, divided in 43 classes. The GTSRB images were taken under different lighting and weather conditions, making TSR a challenging task.

\subsection{Experimental setup}

The implementation of a traditional two stage TSR method will serve as a base for comparing our proposed method. In this paper, the proposed method was tested using two commonly used set of features: grayscale intensity levels and Histograms of Oriented Gradients.

Grayscale intensity levels (GRAY) were used to accurately recognize faces in the eigenfaces method proposed in Ref.20. In our experiments, all traffic sign images were scaled using bilinear interpolation to a fixed size of $28 \times 28$ pixels as in Ref.21, resulting in 784 features.

The Histograms of Oriented Gradients (HOG) features were firstly proposed by Dalal \& Triggs. ${ }^{[6]}$ This popular set of features has already been used for TSR, as mentioned in the Related Work section, giving accurate results. In our experiments, the HOG features were computed using a fixed size of $40 \times 40$ pixels in all images, cell sizes of $5 \times 5$ pixels, and block sizes of $10 \times 10$ pixels, with 8 signed orientation bins, as in Ref.5. The result is a set of 1,568 features.

Considering the large amount of generated features, we used Linear Discriminant Analysis (LDA) ${ }^{[9]}$ for dimensionality reduction. As a result, both training sets (GRAY and HOG) are composed by only 42 features. In order to guarantee a straight and fair comparison between the traditional approach and the proposed method, all classification experiments were conducted using the $k$-NN classifier, with $k=3$ neighbors, using the implementation provided in WEKA. ${ }^{[22]}$

\subsection{Balancing the training set}

Our hypothesis is that applying balancing algorithms helps improving minority class recognition in TSR. In order to prove this hypothesis, two different balancing algorithms were applied following our proposed method: 1) Resampling (RES) minority class training cases using a resampling size value of $300 \%$, as suggested in Ref.2; and 2) SMOTE, that was applied several times, once for every minority class, using the imbalance ratio as percentage of new objects to be generated.

Table 1 shows the resulting number of training cases after applying the balancing algorithms to the original training set, as well as the resulting imbalance ratio and the global accuracies for recognition. It can be seen that, by applying RES and SMOTE algorithms, a good balanced training set can be generated.

Table 1: Number of instances of the training set, after applying balancing algorithms; imbalance ratio and Acc (global recognition accuracy).

\begin{tabular}{llll}
\hline Description & Training cases & $\begin{array}{l}\text { Imbalance } \\
\text { Ratio (IR) }\end{array}$ & Acc (\%) \\
\hline GRAY & 39209 & 10.71 & 90.10 \\
GRAY+RES & 117627 & 1.08 & 89.45 \\
GRAY+SMOTE & 81923 & 5.29 & 89.96 \\
HOG & 39209 & 10.71 & 95.28 \\
HOG+RES & 117627 & 1.08 & 94.76 \\
HOG+SMOTE & 81923 & 5.29 & 95.20 \\
\hline
\end{tabular}

Once the new training sets were created by using balancing algorithms, the next step was to apply a classifier. Figures 2 and 3 show the accuracy per class for each training set: original, balanced using RES, and balanced using SMOTE. Both figures plot the number of training cases per class, in order to know the recognition rate improvement of every class when using each different training set.

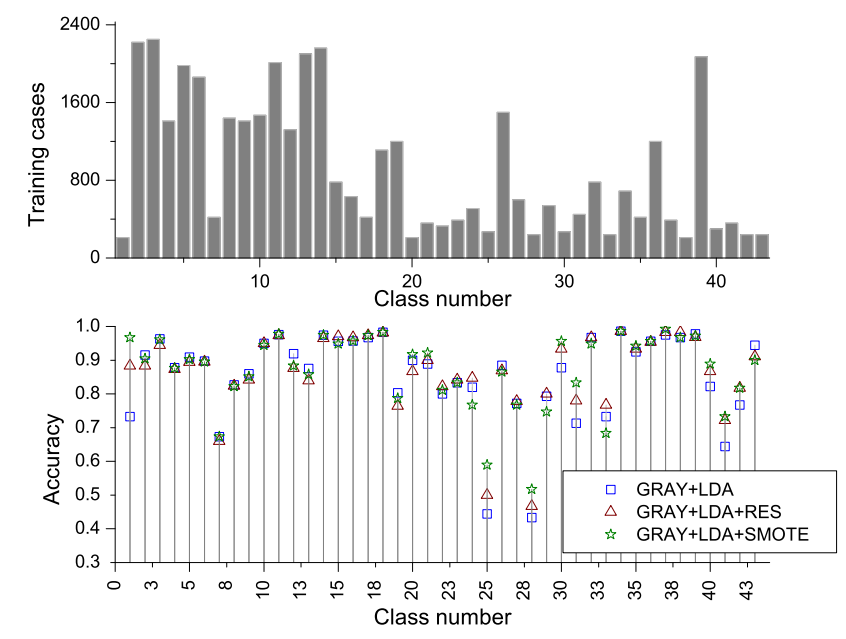

Figure 2: Up) Number of training cases per class. Down) Accuracies per class for gray features.

Figure 2 shows the recognition rates per class when GRAY features were used for classification. Minority classes 1, 25, 30 , and 41 present important improvements in their individual recognition rates, compared to the traditional method. On the contrary, some majority classes like 2 and 3 present a slight decrease in their recognition accuracies. However, in Table 1, it can be seen that global recognition accuracies for these set of features remain almost unchanged.

Figure 3 plots the recognition rates per class when HOG features were used as attributes. Similarly, some minority classes like 1 and 31 present considerable improvements in their recognition rates, while some majority classes like 12 
or 19 showed a slight reduction in their accuracies. Again, from Table 1, we can observe a very small variation in the global recognition accuracies.

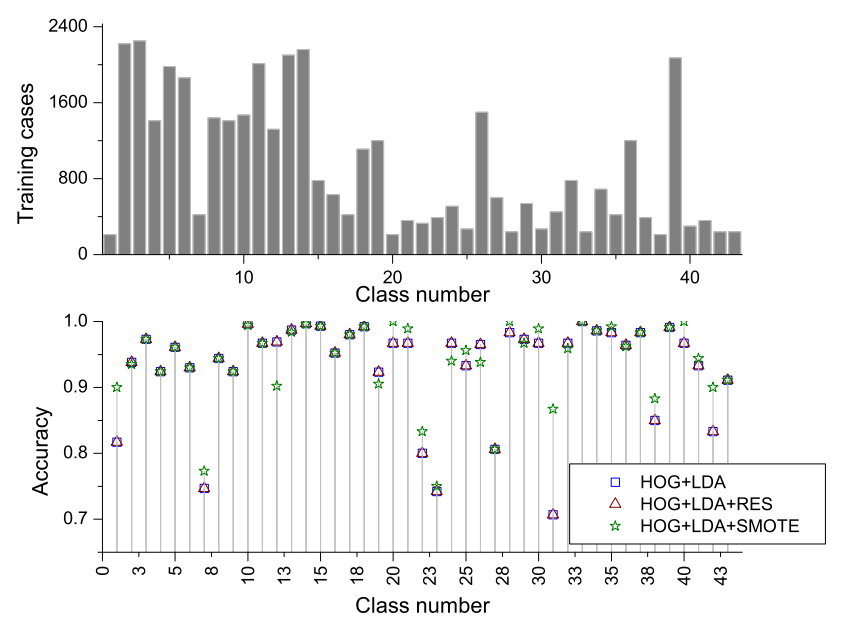

Figure 3: Up) Number of instances per class. Down) Accuracies per class for HOG features.

\section{Conclusions}

This paper proposes a new method for Traffic Sign Recognition, where the inherently imbalanced training sets and their negative effects on classifiers were tackled by applying balancing algorithms. The motivation of this work was to improve of the recognition rates of minority classes, without seriously affecting global accuracies.

In our experiments, two popular feature sets, which have already been used for TSR were tested: Grayscale intensity levels (GRAY) and Histograms of Oriented Gradients (HOG). In addition, two different balancing algorithms: resampling minority class training cases (RES) and SMOTE were used for balancing the training set. Experimental results show that, in spite of the features used and the balancing algorithm applied, our proposed method helps improving recognition rates of minority classes, while global accuracies remain high.

In the future, we will apply this new method on traffic sign detection tasks, in order to minimize false positives and miss-classifications.

\section{Acknowledgements}

This work was partly supported by the University of Guanajuato and DAIP-UG under the Call for Institutional Research 2014 by the project $437 / 2014$; by the National Council of Science and Technology of Mexico (CONACyT) through the project grants CB2008-106443 and CB2008106366; and the scholarship grant 229696.

\section{References}

[1] Ganganwar V. An overview of classification algorithms for imbalanced datasets. International Journal of Emerging Technology and Advanced Engineering. 2012; 2(4): 42-47.

[2] Hernandez J, Carrasco-Ochoa JA, Martinez-Trinidad JF. An Empirical Study of Oversampling and Undersampling for Instance Selection Methods on Imbalance Datasets. In: Progress in Pattern Recognition, Image Analysis, Computer Vision and Applications; 2013. p. 262-269.

[3] Mathias M, Timofte R, Benenson R, Van Gool L. Traffic sign recognition - How far are we from the solution? In: Neural Networks (IJCNN), The 2013 International Joint Conference on; 2013. p. 1-8.

[4] Mogelmose A, Trivedi MM, Moeslund TB. Traffic Sign Detection and Analysis: Recent Studies and Emerging Trends. In: Intelligent Transportation Systems (ITSC), 2012. 15th Inernational IEEE Conference on; 2012. p. 1310-1314.

[5] Stallkamp J, Schipsing M, Salmen J, Igel C. Man vs. computer: Benchmarking machine learning algorithms for traffic sign recognition. Neural Networks. 2012; 32: 323-332. http://dx.doi.org /10.1016/j.neunet. 2012.02.016

[6] N. Dalal and B. Triggs. Histograms of oriented gradients for human detection. Computer Vision and Pattern Recognition, IEEE Computer Society Conference on, vol. 1, 2005, pp. 886-893.

[7] G. Overett and L. Petersson, "Large scale sign detection using HOG feature variants," in Intelligent Vehicles Symposium (IV), 2011 IEEE, 2011, pp. 326-331.

[8] F. Zaklouta, B. Stanciulescu, and O. Hamdoun. "Traffic sign classification using K-d trees and Random Forests. Neural Networks (IJCNN), The 2011 International Joint Conference on, 2011, pp. 2161-4393.
[9] Herve Abdi and Lynne J. Williams. Principal Component Analysis. Wiley Interdisciplinary Reviews: Computational Statistics. 2010; 2(4): 433-459. http://dx.doi.org/10.1002/wics. 101

[10] Miroslav Kubat and Stan Matwin. Addressing the curse of imbalanced training sets: one-sided selection in ICML. 1997; 97: 179186.

[11] D. Ciresan, D. Meier, J. Masci, and J. Schmidhuber. A committee of neural networks for traffic sign classification in Neural Networks (IJCNN), The 2011 International Joint Conference on, 2011, pp. 1918-4393.

[12] P. Sermanet and Y. LeCun. Traffic sign recognition with multi-scale convolutional networks in Neural Networks (IJCNN). The 2011 International Joint Conference on, 2011, pp. 2809-2813.

[13] Nitesh V. Chawla, Kevin W. Bowyer, Lawrence O. Hall, and W. Philip Kegelmeyer. SMOTE: synthetic minority over-sampling technique. Journal of Artificial Intelligence Research. 2002; 16: 321357.

[14] Ivan Tomek. Two Modifications of CNN. Systems, Man and Cybernetics, IEEE Transactions on, vol. SMC-6, no. 11, pp. 769-772, 1976.

[15] Inderieet Mani and I. Zhang. kNN approach to unbalanced data distributions: a case study involving information extraction in Proceedings of Workshop on Learning from Imbalanced Datasets, 2003.

[16] Shizhao Zhang. KNN-CF Approach:Incorporating Certainty Factor to KNN Classification. IEEE Intelligent Informatics Bulletin. 2010; 11(1): 24-33.

[17] Songbo Tan. Neighbor-weighted k-nearest neighbor for unbalanced text corpus. Expert Sysems with Applications. 2005; 28(4): 667671. http://dx.doi.org/10.1016/j.eswa.2004.12.023

[18] Gustavo Batista, Ronaldo Prati, and Maria Carolina Monard. A study of the behaviour of several methods for balancing machine 
learning training data. SIGKDD Explor. Newsl. 2004; 6(1): 20-29. http://dx.doi.org/10.1145/1007730.1007735

[19] Sofia Visa. Issues in mining imbalanced data sets - a review paper in in Proceedings of the Sixteen Midwest Artificial Intelligence and Cognitive Science Conference, 2005, pp. 67-73.

[20] Matthew Turk and Alex Pentland. Eigenfaces for recognition. Journal of Cognitive Neuroscience. 1991; 3(1): 71-86. PMid:23964806 http://dx.doi.org/10.1162/jocn.1991.3.1.71

[21] Sheila Esmeralda Gonzalez-Reyna, Juan Gabriel Avina-Cervantes, and Sergio Eduardo Ledesma-Orozco. Eigen-Gradients for Traffic Sign Recognition. Mathematical Problems in Engineering. 2013.

[22] Mark Hall et al. The WEKA Data Mining Software: An Update. SIGKDD Explor. Newsl. 2009; 11(1): 1931-0145.

[23] S. Maldonado-Bascon, J. Acevedo-Rodriguez, S. Lafuente-Arroyo, A. Henrandez-Caballero, and F. Lopez-Ferreras. An optimization on pictogram identification for the road-sign recognition task using SVMs. Computer Vision and Image Understanding. 2010; 114(3): 1077-3142. 